\section{Un programa escolar para el control del dengue en Honduras: del conocimiento a la práctica}

\author{
Gustavo Adolfo Ávila Montes, ${ }^{1}$ \\ Roxana Araujo, ${ }^{1}$ Elli Leontsini, ${ }^{2}$ \\ Gabriel Orellana Herrera ${ }^{1}$ \\ y Eduardo Fernández Cerna ${ }^{3}$
}

Forma de citar: Ávila Montes GA, Araujo R, Leontsini E, Orellana Herrera G, Fernández Cerna E. Un programa escolar para el control del dengue en Honduras: del conocimiento a la práctica. Rev Panam Salud Publica. 2012;31(6):518-22.

\section{SINOPSIS}

Se describe el Programa Escolar Ambiental (PEA), una iniciativa de control de dengue enfocada en el ámbito de la escuela primaria durante el período de 2005-2010 en varias ciudades de Honduras. El programa fue diseñado para generar mayor conocimiento y destreza en la identificación y el control de criaderos de Aedes aegypti dentro de un contexto de salud ambiental, que incluía además el manejo del agua y los desechos sólidos. Los resultados en el cambio de comportamiento y en la reducción de los indices larvarios fueron satisfactorios en la mayoría de las escuelas que formaron parte de la experiencia, lográndose la participación de los niños, y también de sus padres y maestros. El PEA resultó efectivo además de la reducción de los indices larvarios, en la promoción de la participación comunitaria en temas ambientales con énfasis en el control de Aedes. La inclusión de estos contenidos educativos en el currículo de educación primaria es una asignatura aún pendiente en Honduras.

Palabras clave: dengue; Aedes; educación ambiental; educación en salud; salud escolar; salud ambiental; Honduras.

\footnotetext{
Secretaría de Salud, Programa Nacional de Dengue, Tegucigalpa, Honduras. La correspondencia se debe dirigir a Gustavo Adolfo Ávila Montes. Correo electrónico: avilagus62@gmail.com

2 The Johns Hopkins University, Bloomberg School of Public Health, International Health Department, Baltimore, Maryland, Estados Unidos de América.

McMaster University, Department of Clinical Epidemiology and Biostatistics, Hamilton, Ontario, Canadá
}

El dengue se ha convertido en un muy preocupante problema de salud pública en las Américas. Su incidencia aumentó de unos 2667000 y 58420 casos de dengue y de dengue grave - respectivamente- en el decenio 1990-1999 (1) a unos 8440250 y 221040 en 2000-2010, respectivamente (2). En Honduras, el dengue y el dengue grave han registrado una tendencia que los llevó de unos 95470 y 500 casos —respectivamente- en 1991-2000 a unos 231750 y 8400 en el período 2001-2010 (3). Esta situación ha provocado que la ocurrencia de brotes epidémicos sea cada vez más frecuente, adoptando en la última década un patrón cíclico cada tres a cuatro años (4). En 2010 tuvo lugar la epidemia de mayor magnitud en el país en los últimos 30 años, con 66814 casos de dengue, 3268 de dengue grave y 83 muertes (5). La mayoría de los esfuerzos para controlar el vector han estado dirigidos a la aplicación de adulticidas y larvicidas por personal institucional con escasa participación de la comunidad.

Diversos autores han observado que la adquisición de conocimiento per se no necesariamente conduce a la realización de buenas prácticas $(6,7)$ y que en el caso del control del dengue es necesario rediseñar estrategias innovadoras que fomenten la participación de la comunidad en forma sostenible (8). Una de estas iniciativas ha sido la elaboración de cursos especiales dirigidos a niños de educación primaria con el propósito de que adquieran conocimientos y destrezas que lleven a lograr la adopción de buenas prácticas de control del vector a nivel domiciliar (9-13).

A principios de la década de los noventa Honduras fue el centro de una experiencia de control del Aedes aegypti basada en la participación comunitaria $(4,14,15)$. Una de las contribuciones de este proyecto fue la preparación de un material educativo dirigido a grados superiores de educación primaria denominado Modulo de Higiene Doméstica y Salud Ambiental, cuyo propósito era la adquisición de conocimientos y prácticas de control y eliminación de criaderos por parte de escolares y jefes de familia (16).

En 2002 Ávila y colaboradores evaluaron la efectividad de este módulo y encontraron que había resultado muy útil para promover la participación del núcleo familiar en la reducción de fuentes de Aedes (12). El presente trabajo describe la experiencia de seis años de implementación continua del Programa Escolar Ambiental (PEA) contra el dengue en Honduras. Sus objetivos son determinar y valorar los resultados obtenidos y las lecciones aprendidas del Programa durante el período 2005-2010. 


\section{Descripción de la experiencia}

Inicialmente se seleccionaron siete de ocho ${ }^{4}$ centros escolares de educación primaria pertenecientes al área geográfica del Centro de Salud Tres de Mayo, en la ciudad de Comayagüela. El área de estudio comprendió 10 comunidades urbano marginales que disponen de servicios básicos de agua, electricidad y teléfono, con un total de 6740 viviendas y 36803 habitantes de nivel socioeconómico bajo.

$\mathrm{Al}$ inicio del año lectivo 2005 se brindó un curso de capacitación de dos días dirigido a los maestros de $5^{\circ}$ y $6^{\circ}$ grados de escuela primaria y al personal de salud responsable del acompañamiento técnico. En total recibieron entrenamiento 432 escolares, 12 maestros, 3 técnicos en salud ambiental y 14 bachilleres en salud. Los docentes fueron entrenados en la realización de visitas domiciliarias de inspección larvaria a las viviendas de los escolares, mediante un muestreo por conveniencia (329 viviendas fueron visitadas el primer año). Se utilizó el formulario de supervisión infantil, un instrumento diseñado para los escolares que tiene dibujos de los diferentes depósitos preferenciales del vector (p. ej. barril, botellas, latas y pilas), en donde el escolar registraba la presencia de formas inmaduras de Aedes en estos depósitos, después de haber inspeccionado la vivienda.

En 2010, este programa ya incluía 41 escuelas a nivel nacional, 94 maestros, 4081 escolares y 71 técnicos en salud ambiental, y beneficiaba directamente a unas 21800 personas. Además, se había logrado que el Programa Nacional de Dengue incorporara esta actividad en su Plan Operativo Anual.

\section{La visita de inspección domiciliar}

La actividad central del PEA era la visita de inspección domiciliar. Se dotó a los escolares de chalecos color verde con una leyenda en la parte posterior que identificaba a cada uno como "Vigilante Ambientalista". Se realizaban dos visitas durante el período de la temporada lluviosa con el fin de monitorear el cambio en los índices larvarios. Cada equipo de inspección estaba integrado por cuatro o cinco escolares, el maestro y/o el técnico de salud ambiental o el estudiante de bachillerato, quienes realizaban la detección, identificación y registro de los criaderos en las viviendas de los escolares. Al final de la visita los hallazgos eran reportados por los niños a las madres, indicándoles entonces las medidas necesarias para mantener la vivienda libre de criaderos.

Esta visita domiciliar propiciaba un espacio de enseñanza y práctica entre el maestro, el personal de salud, el escolar y el jefe de familia, donde se reflexionaba sobre los hallazgos realizados y las decisiones pertinentes destinadas a la adopción de buenas prácticas para la prevención del dengue, diferenciándose de la visita de inspección entomológica tradicional (cuadro 1). El impacto de esta intervención durante el período revisado

\footnotetext{
4 Se excluyó una escuela por razones de seguridad.
}

se reflejó en la reducción —estadísticamente significativa- de los índices larvarios clásicos (cuadro 2).

\section{Coordinación intersectorial}

En el comienzo se gestionó el apoyo de la Fundación Vida y la Fundación para la Educación Ricardo Ernesto Maduro Andreu, las cuales aportaron fondos para la compra de materiales educativos e incentivos para la participación de los escolares y padres de familia en actividades de proyección comunitaria. También se concertó que estudiantes del Bachillerato en Salud Comunitaria del Instituto Superación San Francisco realizaran su práctica profesional supervisada en el PEA durante un período de tres meses.

En 2006 se obtuvo una subvención de 4267 euros de la Empresa t-gestiona del Grupo Telefónica de España, producto de que esta experiencia fue escogida como mejor proyecto de tesis del Diploma Superior en Pediatría Tropical edición 2006, donde fue alumno uno de los autores del presente trabajo.

A raíz de los resultados logrados en la reducción de los índices entomológicos y la obtención de reconocimientos en el país (Premio Nacional del Ambiente 2007) e internacionales que calificaron a este programa como la mejor "Experiencia de Buenas Prácticas en Promoción de la Salud en el Ámbito Escolar a nivel de Educación Primaria" (categoría medio ambiente), en los concursos iberoamericanos realizados en 2007 y 2009 (17-18), se consiguió el patrocinio de UNICEF, que desde 2009 ha financiado la implementación del programa y la expansión a ocho ciudades de la nación. Al mismo tiempo, se están llevando a cabo reuniones de abogacía con las autoridades de la Secretaría de Educación y con la Comisión de Educación del Congreso Nacional para que en el mediano plazo se logre introducir el PEA dentro del currículo de educación primaria.

\section{Discusión}

La implementación del PEA en el período 20052010 ha demostrado cambios en la adopción de buenas prácticas en las familias expuestas que llevaron a reducciones significativas de los índices larvarios. Las prácticas de control de criaderos más reportadas fueron referidas al aseo del barril o la pila con "la untadita", un método de desinfección con acción ovicida sobre $A$. aegypti y que ha sido ampliamente descrito $(15,19-21)$. Dado que las actividades se desarrollaron en los meses de mayor incidencia de la enfermedad (época lluviosa), cuando los factores ambientales son más favorables para la transmisión del dengue, se considera que la reducción de los índices obedeció al efecto directo de la intervención educativa realizada a través de los Vigilantes Ambientalistas.

Los resultados de esta experiencia, y lo informado por otros autores $(11,22,23)$, prueban que cuando los programas contra el dengue incluyen un componente de educación dirigido a los escolares generan beneficios porque aumentan el interés y las 
CUADRO 1. Diferencias observadas entre la visita de supervisión infantil y la visita de inspección entomológica para el control del dengue, Honduras, 2005-2010

\begin{tabular}{lll}
\hline \multicolumn{1}{c}{ Variable } & \multicolumn{1}{c}{ Visita de supervisión infantil } & \multicolumn{1}{c}{ Visita de inspección entomológica } \\
\hline Propósito & $\begin{array}{c}\text { Búsqueda de criaderos, educación/ } \\
\text { concientización sobre saneamiento }\end{array}$ & Vigilancia entomológica, intervenciones efectivas \\
Supervisor de la vivienda & Muestreo por conveniencia & Técnico de salud ambiental \\
Selección de la muestra & Hoja de supervisión infantil & Muestreo sistemático \\
Instrumento de recolección de datos & Pre y post-intervención educativa & Formulario entomológico \\
Periodicidad & Número de viviendas positivas y negativas & Trimestral, post-intervención \\
Datos registrados & Maestro, técnico de salud ambiental & Técnico de salud ambiental \\
Supervisión de campo & Vigilancia activa de criaderos potenciales & Control/eliminación de criaderos \\
Práctica esperada & &
\end{tabular}

Fuente: elaboración de los autores.

CUADRO 2. Índices larvarios registrados en las visitas domiciliarias de inspección, por año lectivo. Programa Escolar Ambiental, Honduras, 2005-2010

\begin{tabular}{|c|c|c|c|c|c|c|c|c|c|c|}
\hline \multirow[b]{2}{*}{ Año lectivo } & \multicolumn{5}{|c|}{ Primera visita (pre-intervención) } & \multicolumn{5}{|c|}{ Segunda visita (post-intervención) } \\
\hline & $\begin{array}{l}\text { Viviendas } \\
\text { inspeccio- } \\
\text { nadas } \\
(n)^{\mathrm{a}}\end{array}$ & $\begin{array}{l}\text { Viviendas } \\
\text { positivas } \\
\text { (No.) }\end{array}$ & $\begin{array}{c}\text { Recipientes } \\
\text { positivos } \\
\text { (No.) }\end{array}$ & $\begin{array}{l}\text { Índice de } \\
\text { vivienda }\end{array}$ & $\begin{array}{c}\text { Índice de } \\
\text { Breteau }\end{array}$ & $\begin{array}{l}\text { Viviendas } \\
\text { inspeccio- } \\
\text { nadas } \\
(n)\end{array}$ & $\begin{array}{l}\text { Viviendas } \\
\text { positivas } \\
\text { (No.) }\end{array}$ & $\begin{array}{c}\text { Recipientes } \\
\text { positivos } \\
\text { (No.) }\end{array}$ & $\begin{array}{l}\text { Índice de } \\
\text { vivienda }\end{array}$ & $\begin{array}{c}\text { Índice de } \\
\text { Breteau }\end{array}$ \\
\hline $2005^{b}$ & 282 & 75 & 325 & 26,5 & 115,2 & 329 & 11 & 16 & 3,3 & 4,8 \\
\hline $2006^{b}$ & 167 & 60 & 115 & 35,9 & 68,8 & 168 & 11 & 19 & 6,5 & 11,3 \\
\hline $2007^{b}$ & 416 & 88 & 114 & 21,1 & 27,4 & 415 & 19 & 32 & 4,5 & 7,7 \\
\hline $2008^{b}$ & 583 & 148 & 229 & 38,9 & 57,0 & 583 & 30 & 51 & 19,6 & 36,6 \\
\hline $2009^{b}$ & 132 & 39 & 116 & 29,5 & 87,8 & 97 & 10 & 60 & 10,3 & 61,8 \\
\hline $2010^{c}$ & 112 & 39 & 68 & 34,8 & 60,7 & 84 & 3 & 4 & 3,5 & 4,7 \\
\hline Total & 1412 & 419 & 911 & 29,6 & 64,5 & 1358 & $106^{d}$ & 228 & $7,8^{\mathrm{e}}$ & 16,7 \\
\hline \multicolumn{11}{|c|}{$\begin{array}{l}\text { Fuente: elaboración de los autores. } \\
\text { a El número de viviendas inspeccionadas por año no fue constante debido a limitantes en el personal operativo disponible para realizar las visitas domiciliarias. } \\
\text { b Estos datos corresponden a las escuelas del Distrito Educativo } 7 \text { de Comayagüela. } \\
\text { c Estos datos corresponden a escuelas intervenidas de Danli y San Pedro Sula únicamente. } \\
\text { d El chi cuadrado realizado para el total de viviendas (positividad de viviendas) pre y post fue de } 215,517 \text { y generó un valor } P<0,001 \text { estadísticamente significativo. } \\
\text { e T-test para una sola muestra (muestras pareadas). Se incluyen los índices de vivienda de cada año, pre y post, con resultados de } t=342,16 \text { y } P<0,001 ; \text { para el índice } \\
\text { de Breteau se dio una } t=56,88 \text { para un valor } P \text { de } 0,001 \text {. }\end{array}$} \\
\hline
\end{tabular}

destrezas sobre el control del vector por parte de los niños y los maestros y - por transferencia- en todo el núcleo familiar (figura 1).

En Argentina, actividades similares a la aquí descrita demostraron que los niños constituyeron un complemento fundamental de la acción educativa en salud individual, familiar y comunitaria para participar activamente en el control de la tuberculosis y en la vigilancia entomológica comunitaria de la enfermedad de Chagas $(24,25)$. En este tipo de iniciativas se asegura la integración del enfoque de "abajo arriba" (26), donde la comunidad toma la iniciativa y la responsabilidad en las acciones de control, en contraste con los programas verticales donde ambas corresponden al personal de salud, en tanto la comunidad asume un rol pasivo. De hecho, la presente experiencia se aproxima más al esquema de desarrollo comunitario (27-30), en el cual el objeto no es solo el control del $A$. aegypti sino también el desarrollo de la comunidad y el saneamiento del medio.

La experiencia no estuvo exenta de limitaciones. Por ejemplo, el conflicto suscitado entre el sector magisterial y el gobierno, que obstaculizó la implementación adecuada del programa - particularmente en las ciudades de Tegucigalpa y San Pedro Sula- y en
2008 y 2009 provocó que la reducción de los índices de Aedes no fuera tan acentuada; otras limitaciones fueron la falta de fondos suficientes para financiar la reproducción del material educativo y que no se realizaran investigaciones cualitativas sobre la aceptabilidad de los padres y maestros tal como se realizó en el 2002 (12). En el mismo sentido, la falta de decisión y voluntad política de las autoridades de salud y de educación ha sido la principal barrera que ha impedido que este programa trascienda de una experiencia piloto a convertirse en un contenido pedagógico oficial en el currículo de educación primaria.

Por otra parte, la coordinación intersectorial ha sido una estrategia muy eficaz en la lucha anti vectorial y, al momento de preparar el presente trabajo, un factor clave para que el PEA se haya implementado en forma continua (31). Otros países de América Latina también han realizado esfuerzos para controlar hábitats vectoriales. En Panamá y Puerto Rico, sociedades de servicios como los clubes de rotarios han hecho aportes valiosos en términos de financiamiento, abogacía y mensajes de servicio público para las campañas comunitarias de reducción de fuentes de Aedes $(28,32)$. En Colombia, la Secretaría de Salud de Bucaramanga y la Corporación Tres-H del Club 
FIGURA 1. Modelo de impacto simplificado de cómo la implementación del programa escolar para controlar el dengue conduce a la adopción de buenas prácticas

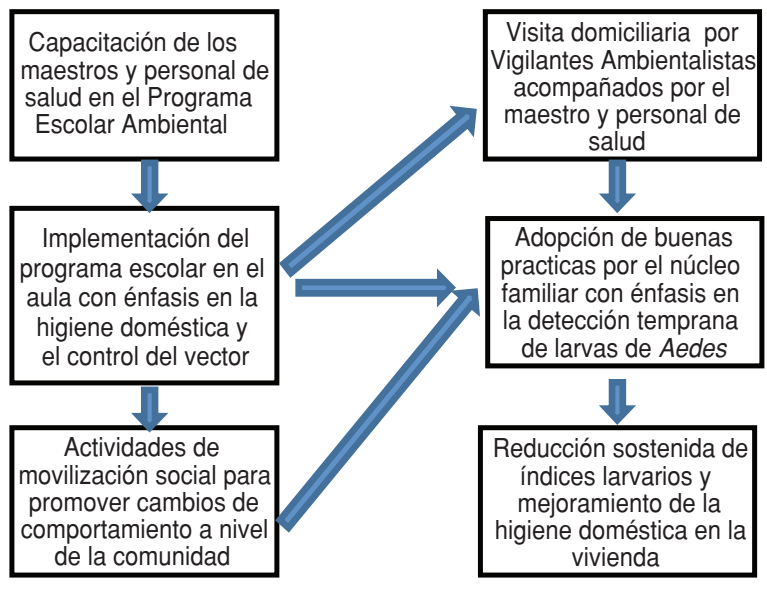

Fuente: elaboración de los autores.

Rotario de Bucaramanga-Chicamocha, promovieron la movilización masiva de estudiantes, jefes de familia y grupos comunitarios, logrando reducciones sostenidas de los índices larvarios (33). Winch y colaboradores describen este tipo de programas como un nuevo paradigma, donde el Estado asume una función de coordinación y supervisión dentro de una coalición de grupos y organizaciones, cada uno con funciones específicas relacionadas con el control del vector del dengue (34).

En el caso de Honduras, el PEA se transformó en una herramienta complementaria a las acciones regulares del Programa Nacional de Dengue, que facilitó una transferencia efectiva de conocimientos desde el aula hacia la vivienda del escolar, con la cual se consiguió de manera pasiva sensibilizar a los jefes de familia acerca del problema del dengue y motivarlos a actuar concretamente para controlar el vector. No obstante, es necesario que quienes trabajan en esta clase de iniciativas incentiven enfáticamente la participación de otros agentes de los sectores público y privado y de la sociedad civil, incluyendo autoridades de las secretarías de educación y salud.

Finalmente, hará falta llevar a cabo estudios cualitativos para determinar la aceptabilidad social del PEA entre las madres y padres de familia, los docentes y el personal de salud. El programa aquí descrito demostró que es una herramienta idónea para promover activamente la participación decidida de la comunidad en el control vectorial del dengue y que además puede contribuir a la mejora del saneamiento ambiental de las comunidades. La inclusión de estos contenidos educativos en el currículo de educación primaria es una tarea todavía pendiente de cumplimiento en Honduras.

\section{SYNOPSIS}

\section{A school program for dengue control in Honduras: from knowledge to action}

The Environmental School Program (PEA, for its Spanish acronym), a dengue control initiative focused on primary schools that took place during 2005-2010 in several cities in Honduras, is described. The environmental health program was designed to increase knowledge and develop skills in the identification and control of Aedes aegypti breeding sites, as well as in water and solid waste management. The results, as measured by behavioral change and reduced larval indices, were satisfactory in the majority of the participating schools. The initiative involved not only children, but also their parents and teachers. In addition to reducing larval indices, PEA was successful in promoting community participation in environmental issues, particularly Aedes control. The inclusion of this educational content in the primary school curriculum in Honduras remains pending.

Keywords: dengue; Aedes; environmental education; health education; school health; environmental health; Honduras.

\section{REFERENCIAS}

1. San Martín JL, Brathwaite $\mathrm{O}$, Zambrano B, Solórzano JO, Bouckenooghe A, Dayan GH, et al. The epidemiology of dengue in the Americas over the last three decades: a worrisome reality. Am J Trop Med Hyg. 2010;82(1):128-35.

2. Guzmán $M G$, Brathwaite $O$, Enria $D$, Hunsperger E, Kouri G, Montoya R, et al. Dengue in the American Region. An update. Disponible en: http:// blog.tropika.net/tropika/files/2011/ 07 /dengue-by-guzman-et-al-jun 2011.pdf Acceso el 28 de marzo de 2012.

3. Boletin Semanal de Enfermedades de Notificación Obligatoria. Dirección de
Vigilancia de la Salud, Secretaria de Salud, Tegucigalpa, Honduras.

4. Ávila Montes GA, Araujo R, Orellana Herrera G. Situación epidemiológica del dengue en Honduras período 1991-2010. Rev Med Hondur. 2010;78(3):156-62.

5. Pan American Health Organization. Información regional dengue: número de casos 2010. Disponible en: http://new. paho.org/hq/index.php?option= com docman\&task=doc download \&gid=13075\&Itemid $=$ Acceso el 28 de marzo de 2012.

6. Khun S, Manderson L. Community and school-based health education for dengue control in rural Cambodia: a pro- cess evaluation. PLoS Negl Trop Dis. 2007;1(3):e143.

7. Cordero Conejo AC, Sandí Nogueral F, Mejías Fonseca G, Silva Silva RA, Campos Rodríguez MV, Soto L, et al. Reseña de una experiencia de promoción de la salud y lucha contra el dengue utilizando el control biológico, en escuelas de Chacarita, Puntarenas, Costa Rica. Revista Costarricense de Salud Pública. 2000;9(17):20-5.

8. San Martín JL, Brathwaite-Dick O. La estrategia de gestión integrada para la prevención y el control del dengue en la región de las Américas. Rev Panam Salud Publica. 2007;21(1):55-63. 
9. Soto Henández RJ, Fernández Cerna EA, Ávila Montes GA. Evaluación de un programa educativo sobre dengue y Aedes aegypti focalizado en niños de escuela primaria. Rev Med Hondur. 1995; 63(1):12-8.

10. Winch PJ, Leontsini E, Rigau-Pérez JG, Ruiz-Pérez M, Clark GG, Gubler DJ. Community-based dengue prevention programs in Puerto Rico: impact on knowledge, behavior, and residential mosquito infestation. Am J Trop Med Hyg. 2002;67(4):363-70.

11. Vivas E, Guevara de Sequeda M. Un juego como estrategia educativa para el control de Aedes aegypti en escolares venezolanos. Rev Panam Salud Publica. 2003;14(6):394-401.

12. Ávila Montes GA, Martínez M, Sherman C, Fernández Cerna E. Evaluacion de un módulo escolar sobre dengue y Aedes aegypti dirigido a escolares en Honduras. Rev Panam Salud Publica. 2004;16(2):84-94.

13. Núñez-Samudio V. Niños contra el dengue. An Pediatr (Barc). 2007;66(1)89.

14. Leontsini E, Gil E, Kendall C, Clark GG. Effect of a community-based Aedes aegypti control programme on mosquito larval production sites in El Progreso, Honduras. Trans R Soc Trop Med Hyg. 1993;87(3):267-71.

15. Fernández EA, Leontsini $E$, Sherman $C$ Chan AST, Reyes CE, Lozano RC, et al. Trial of a community-based intervention to decrease infestation of Aedes aegypti mosquitoes in cement washbasins in El Progreso, Honduras. Acta Trop. 1998;70(2):171-83.

16. Fernández EA, Reyes $\mathrm{CE}$, Hernández D. Módulo escolar higiene doméstica y salud ambiental. San Pedro Sula, Honduras: Editorial Capiro; 1997.

17. Instituto de Nutrición de Centro América y Panamá. I Concurso Iberoamericano de Buenas Prácticas en Promoción de la Salud en el Ámbito Escolar. Disponible en: http://www. sica.int/busqueda/Noticias.aspx?ID Item $=17905 \&$ IDCat $=3 \& \operatorname{IdEnt}=29 \&$ $\mathrm{Idm}=1 \&$ IdmStyle $=1$ Acceso el 28 de marzo de 2012

18. Organización Panamericana de la Salud. Biblioteca Virtual de Desarrollo Sostenible y Salud Ambiental (BVSDE). Segundo
Concurso Iberoamericano de Buenas Prácticas en Promoción de la Salud en el Ámbito Escolar. Disponible en: http:// www.bvsde.ops-oms.org/bvsdeescuelas/ concurso/ganadoresinternac.html Acceso el 28 de marzo de 2012.

19. Sherman C, Fernández EA, Chan AS, Lozano RC, Leontsini E, Winch P. La Untadita: A procedure for maintaining washbasins and drums free of Aedes aegypti based on modification of existing practices. Am J Trop Med Hyg. 1998; 58(2):257-62.

20. Méndez J, Fernández EA. Taller sobre avances recientes en el control del $\mathrm{Ae}$ des aegypti basado en la comunidad: Honduras y México. Mérida, Yucatán, México, D.F.: Secretaría de Salud de México; 1996.

21. Chan AS, Sherman C, Lozano RC, Fernández EA, Winch PJ, Leontsini E. Development of an indicator to evaluate the impact on a community-based Aedes aegypti control intervention, of improved cleaning of water-storage container by householders. Ann Trop Med Parasitol. 1998:92(3):317-29.

22. Vesga-Gómez C, Cáceres-Manrique FM. Eficacia de la educación lúdica en la prevención de dengue en escolares. Rev Salud Publica. 2010;12(4):558-69.

23. Combina VMV. Determinación del nivel de conocimiento sobre dengue en alumnos de escuelas municipales de la ciudad de Córdoba, Argentina. Perspectivas para la prevención. Rev Salud Publica. 2008;12(1):37-51.

24. Darnaud R, Dato MI. Educación para la salud en escuelas argentinas: concurso de plástica como actividad motivadora. Rev Panam Salud Publica. 2009;25(2): 181-7.

25. Crocco L, Rodríguez C, Catalá S, Nattero J. Enfermedad de Chagas en Argentina: herramientas para que los escolares vigilen y determinen la presencia de factores de riesgo en sus viviendas. Cad Saude Publica. 2005;21(2):646-51.

26. Gubler DJ. Aedes aegypti and Aedes aegypti-borne disease control in the 1990's: top down or bottom up. Charles Franklin Craig Lecture. Am J Trop Med Hyg. 1989;40(6):571-8.

27. Winch PJ, Kendall C, Gubler D. Effectiveness of community participation in vector-borne disease control. Health Policy Plan. 1992;7(4):342-51.

28. Organización Panamericana de la Salud. Dengue y dengue hemorrágico en las Américas: guías para su prevención y control. Washington, D.C.: OPS; 1995. (Publicación científica 548)

29. Vanlerberghe $\mathrm{V}$, Toledo ME, Rodríguez M, Gómez D, Baly A, Benítez JR, et al. Community involvement in dengue vector control: cluster randomised trial. MEDICC Rev. 2010;12(1):41-7.

30. Erlanger TE, Keiser J, Utzinger J. Effect of dengue vector control interventions on entomological parameters in developing countries: a systematic review and meta-analysis. Med Vet Entomol. 2008;22(3):203-21.

31. Parks W, Lloyd L. Planificación de la movilización y comunicación social para la prevención y control del dengue: guía paso a paso. Washington, D.C.: OPS; 2004.

32. Clark GG, Duane J. Gubler DJ, Seda H, Pérez C. Development of Pilot Programmes for Dengue Prevention in Puerto Rico: A Case Study. Dengue Bulletin. 2004;28:48-52. Disponible en: http://www.searo.who.int/LinkFiles/ Dengue_case_study_10__puerto_rico_. pdf Acceso el 28 de marzo de 2012.

33. Luna JE, Chain I, Hernández J, Clark GG, Bueno A, Escalante R, Angarita S, et al. Social mobilization using strategies of education and communications to prevent dengue fever in Bucaramanga, Colombia. Dengue Bulletin. 2004;28:17-21. Disponible en: http://www.searo.who. int/LinkFiles/Dengue_case_study_3 columbia .pdf Acceso el 28 de marzo de 2012.

34. Winch P, Leontsini E, Lloyd L. Mosquito control: behavioral and community interventions in Dengue. London, U.K.: Ed. Halstead S. Tropical Medicine Science and Practice, Volume 5, Imperial College Press; 2008.

Manuscrito recibido el 22 de agosto de 2011. Aceptado para publicación, tras revisión, el 23 de febrero de 2012. 